\section{Analysis of airflow and air volume during continuous speech}

\section{YOSHIYUKI HORII and PAUL A. COOKE Department of Audiology' and Speech Sciences Purdue University, West Lafayette, Indiana 47907}

Program RESPAN is a respirometric analyzer operating on airflow (volume velocity) function during speech obtained through a pneumotachograph-pressure-transducer-amplifier system (Hardy, 1965; Isshiki \& Ringel, 1964). The volume velocity function during speech typically consists of large negative portions corresponding to inhalation and slowly varying positive $\mathrm{dc}$ signals (phonetic and syllabic components) superimposed by higher frequency ac components (voice fundamental frequency for voice segments). Specific functions of the program are (1) to detect inhalation and exhalation, (2) to calculate total inhaled and exhaled air volume for each breath group, (3) to obtain time ratio of contiguous inhalation and exhalation, (4) to generate piecewise linear approximation of the volume velocity function, and (5) to calculate the maximum, minimum, and average rate of air usage for closureto-closure segments (segments between a point of zero volume velocity to next zero volume velocity during speech) within each breath group.

The program performs as a combination of peak detector, valley detector, plateau detector, zero detector, and inhalation detector. Primary programming consideration was given to reject the high-frequency (voice fundamental frequency and quantization noise) components in a peak-yalley-plateau detection and to process multichannel signals of virtually unlimited length. The multichannel processing has been implemented into the program because of frequent needs to simultaneously investigate airflow, air volume, and voice signals. The present program accepts a five-channel data and allows a maximum signal length of about $1 \mathrm{~h}$.

Input/Output. The data collection procedures are illustrated in Figure 1. Channels 1 through 5 are used for voice signals, airflow signals with high-gain amplification, integrated airflow (cumulative volume) signals, the airflow signals with low-gain amplification, and identification signals $(-10 \mathrm{~V}$ dc), respectively. The purpose of the low-pass filtering for flow and volume signals is to minimize voice fundamental frequency components. Signals on the five channels are scanned and quantized through an analog-to-digital converter at 5,000 times/sec, resulting in a $1-\mathrm{KHz}$ sampling rate for each channel. The digitized data are written on a computer magnetic tape through a chaining buffer. Use of the two levels of amplification for airflow signals is motivated for optimal use of dynamic range of the system for both relatively low exhalatory airflow during speech and considerably larger airflow during the inhalation. If a single channel were used to record both inhalation and exhalation, resolution of airflow signal during speech would be considerably reduced. Voice signals on the first channel are used to temporally relate the aerodynamic events with acoustic and articulatory events. Since voice signals are picked up by a microphone inserted incident to air passage in the pneumotachograph, they have predominantly low-frequency components (due to the resonant characteristics of such a system). The purpose of the identification signal of $-10 \mathrm{~V} \mathrm{do}$ on the fifth channel is to allow the program to double-check and align, if necessary, the sequence of the channels for each scan and eliminate any extraneous data from analysis. The integration signal (reset either every second or every $10 \mathrm{sec}$ ) provides cumulative volume measures and is primarily used to compare and validate results of this computer analysis procc dure with those obtained by other techniques that employ such integration methods on volume measurements (Hardy \&

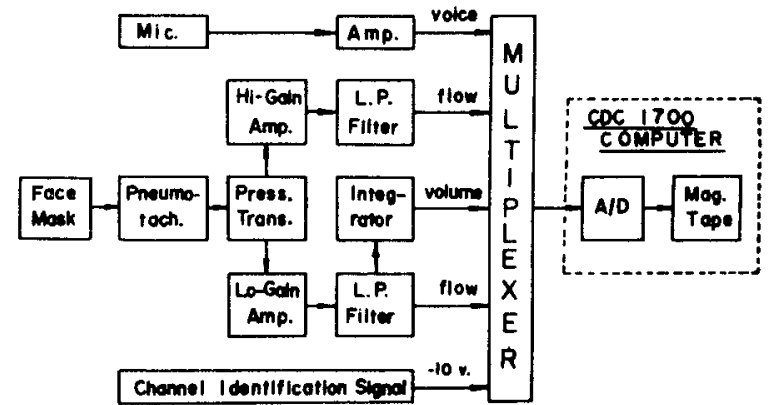

Figure 1. A block diagram illustrating the respirometric datacollection procedures.

Edmonds, 1968; Jacob, Cooke, \& Daniloff, 1973). A multichannel FM tape recorder can be employed to store analog signals for off-line execution.

In addition to the digitized analog signals on the magnetic tape, the program requests several threshold values for airflow signals and a conversion factor through a Teletype. These thresholds are (1) zero level above which the airflow is considercd to be present, (2) noise level and minimum noise duration, which are used to reject voice fundamental frequency components and quantization noise in peak-valley-plateau detection, (3) inhalation level, which flags the presence of inhalation, and (4) minimum plateau duration above which the airflow, if its level stays within the noise level, is considered to be in plateau condition. The conversion factor, on the other hand, is derived by preliminary analysis of calibration airflow signals. The calibration signal with a known volume velocity is analyzed with a conversion factor of 1.0 and the program returns a new conversion factor to be used for the subsequent analysis.

The program accomplishes derivation of all the measurements conventionally obtained by visual inspection and hand measurements of oscillographic records of airflow and volume signals during speech. Statistical data (histograms and the mean and standard deviation of instantaneous volume velocity) are also printed out at the end of analysis. Optionally, the volume velocity function, its piccewise approximation and speech waveform can be displayed on an oscilloscope or on a multichannel graphic level recorder. The program can be employed for analysis of a variety of normal and disordered speech.

Program Language and Computer. The program is written in FORTRAN IV except for two subroutines to digitize and write data on magnetic tape and to read the digitized data on the tape back to core, which is written in assembly language for a CDC 1700 computer.

Availability. Description and listing of the program are available free (except postage) on request. Send requests to Yoshiyuki Horii, Department of Audiology and Speech Sciences, Purdue University, West Lafayette, Indiana 47907.

\section{REFERENCES}

Hardy. J. C. Airtlow and air pressure studies. Proceedings of the conference on communicative problems in cleft palate. ASHA, 1965. Report No. 1. 141-152.

HARDY, J. C.. \& EDMONDS, T. Electronic integrator for measurement of partitions of lung volume. Journal of Speech and Hearing Resear'h, 1468, 11, 777-786.

ISshiki, N., \& Ringei, R. L. Airtlow during the production of selected comsonants. Joumal of Speech and Hearing Research, $1904,7,23,3-244$.

JACOB, C. T. . COOKE. P., \& DANILOFF, R. G. Linguistically related parameters of airflow rate during speech and reading. ASHA, 1473, 15, 440(a). 